\title{
Forward Problem in Electrocardiogram: A Review of Certain Approaches
}

\author{
Gaddam.Chandra Mohan, Dr.Swapna Devi \\ NITTR, sector 26 Chandigarh
}

\begin{abstract}
Dealing the Forward problem in electrocardiogram using various approaches have been presented which are available in published literature. Human torso can be modeled which consist of various tissues of human body. Volume and surface methods like boundary element method, finite element methods based on selecting choice of nodes and meshes construction orientation. . Computer models used for simulation purpose helpful to study the torso effect on heart.
\end{abstract}

Keywords: - Electrocardiogram, Forward problem, human torso, Boundary element method, finite element method, Simulation.

\section{INTRODUCTION}

Electrocardiogram is the interpretation of body surface potentials recorded at the body surface due to the Electrical activity of the heart. The objective of forward and inverse problem is to understand the electrical activity qualitatively and quantitatively. For this purpose we have to use the concept of equivalent source similar to torso. The forward problem hence consist of calculating the potential distribution at the body surface due to the presence of selected equivalent source inside the conductor.

\section{TORSO MODEL}

The forward problem of electrocardiogram related to the electric signal generated in the heart with the resulting potential distribution on the body surface. The electric field in the human torso is mainly determined by the size and position of the internal organs and structures. These are also referred to as inhomogeneties. The various tissues, fluids, and structures are known to vary with respect to their conductivities (1). Hence the mentioned torso model includes all major anatomical structures like blood, lungs, fat, anisotropic skeletal muscle intestine, liver, kidneys, bone, cartilage and spleen. The amplitudes of both atrial and ventricular most sensitive for blood, skeletal muscle conductivity and anisotropy aswell as for heart, fat and lungs. Like, this many factors of different parameters to be analyzed properly for comprehensive features of the ECG signal.

Numerical techniques are required for calculating the forward problem when considering irregularity and complexity of the realistic geometry of the human body. Volume methods like finite element, finite difference, and finite volume methods based on differential equation methods. Surface methods like boundary element method based on the integral function techniques. Generally the volume and surface methods require choice of nodes and construction of meshes.

\section{BOUNDARY ELEMENT MODEL}

In boundary element method only the interfaces between various tissue regions need to be modeled in simulation. The quality of the boundary element discretization affects the accuracy of the numerical solution. Construction of high quality meshes is always time consuming. Adaptive BEM (a BEM) has been developed and validated as an effective method to tackle such problems in electromagnetic and mechanical fields. Much work has not been done in the ECG forward problem as for available published literature. A method known as ha BEM (hierarchical BEM) investigated (2). It produces refined meshes through adaptive adjustment of the elements connection. This method produces better mesh results, accuracy, effective and fast convergent than traditional methods.

\section{FINITE ELEMENT METHOD}

In conventional finite element method (FEM) both node information and element information i.e. the connection between nodes to construct shape functions used for construction of their shape functions. But, in the mesh less FEM only the node distribution within the domain of the problem is required to construct their shape functions (3). These methods are having good convergence than traditional methods. 


\section{3D GRAPHS}

The following steps are required for construction of computer simulation algorithms in ECG forward problem. They are like .constructing heart and torso 3D graphs, simulating the cardiac bioelectric excitation process , Calculating myocardial action potentials and dipoles and calculating body surface potentials etc. The more actual physical model based on the relation between cardiac bioelectric process and body surface potentials. This new scheme of actual action potential model and myocardial excitation vector propagation algorithm serves to reach the specified level to some extent(4).

\section{POLYNOMIAL CHAOS}

The determination of cardiac activity from externally recorded potentials is an inverse problem and proven as a difficult one. This is because of the attenuation property of the potentials in propagation from the heart to the torso surface. Another source of error in these ill posed problems is their high sensitivity to perturbations in the geometry of thorax and electrical conductivity of the tissues of volume conductor. Hence errors in this forward solution exaggerate uncertainties which lead to understanding the relation between geometric and conductivity errors make futile in bioelectric modeling. Model parameters with uncertainties can be treated as statistical distributions which lead to statistical characteristics in stochastic system. Polynomial chaos (PC) is more effective approach to handle systems exhibiting complex interactions among their stochastic parameters (5). PC can be provided higher order statistics over the entire domain and thus a valuable tool for investigating two or three dimensional biological parameters to stochastic parameters. This way of computing approach investigating the effect of multiple stochastic parameters, further it also tracks even multiple random dimensional stochastic parameters in a reasonable amount of time.

\section{CARDIO ELECTRO MECHANICS}

ECG wave form depends on the mechanical motion of the heart. The influence of this motion can be studied through forward modeling including biodomain description and coupled model of cardioelectromechanics (6).It was found that mechanical motion has no significant effect over $\mathrm{T}$ wave morphology. Hence it can says that mechanical motion of the heart will not effect on recovery of ventricular myocardium.

\section{ELECTRICAL IMPEDANCE TOMOGRAPHY}

Another forward model deals with problem of Electrical impedance tomography (EIT) problem (7). This method approach is to compute the potential at electrodes on the body surface, given a set of current patterns injected by those same electrodes and a known conductivity map. And also here, 3D Boundary element method (BEM) solution used which imposes the assumption that the internal organ conductivities are piece wise constant in the volume. This seems to decreases the number of unknowns. In the long term EIT can be used to reconstruct conductivity map which can be used in the inverse problem of electrocardiography. Mathematical models used to get quantitative electrical image from high density ECG recordings.

\section{CELL MODELING}

The process of modeling the electrical activity at the cell (continuum)through the torso surface, while maintaining a continuous path of current flow is have been investigated(8). By this thorough study clinical validation feasibility will be facilitated further use for clinical diagnosis. Realistic model allows to analyzing the abnormal heartbeats (arrhythmias) in cardiac tissue.

\section{FINITE DIFFERENCE METHOD}

Mathematical model of cellular action potential with its underlying ionic currents in human myocardium based (9) is the sophisticated model which is based upon luo-rudy phase model. The activation process is carried out within a three dimensional model taking into account the behavior of each single cell membrane. The propagation is simulated using the finite difference method in the time domain.

\section{COMPUTER SIMULATION MODEL}

The most important application of the forward problem is building computer heart model which can be used for simulation of electrocardiogram (10).Another important application has been to study the effect of torso in homogeneities on the ECG. Further, this forward problem methodology has also been used for reciprocal problem of obtaining the currents due to current sources applied at the body surface. Surface methods uses simple torso models with fewer elements. But underlying integral equations couple the potential at every element to the potential at every other element, the coefficient matrix characterizing the set of equations fully populated. Volume methods use more elements consequently more potentials determined. The potential at each point is expressed only in terms of potentials at its nearest neighbors. 


\section{TESSELLATION SCHEMES}

In the forward solution actual surface potentials, generated by neural sources within the body were calculated. While calculating surface potential some of the factors will affect the accuracy. These are like numerical approximations, constant potential versus linear potential basis functions, sharp edged versus smooth surfaced volumes, source position and orientation, aspects of volume conductor including volume shape, density of surface elements, element shape and effect of refinement shape in the numerical method. A rigorous random test pattern for source location, compare BEM techniques on four different shapes of volume conductors (spherical, prolate and oblate spheroidal and rectangular) and consider special varieties of tessellation schemes can be attempted to understand the factors(11).

\section{SURFACE METHODS}

Simulating cardiac pathologies with computer heart model that incorporates anisotropic Propagation is the major application of forward problem. Hence a overall view of these techniques will give a clarity of approach to deal with these problem in a systematic way (12). Surface methods based on integral equations for the potential derived by green's second identity to the geometry.

\section{CONCLUSION\& FUTURE SCOPE}

The approaches so far deal with the availability of work stations. If more powerful work stations developed there is a scope for developing various models of the torso and consequently more and more accurate solutions of forward problem. It remains to see in future whether this desire to be fulfilled,

\section{ACKNOWLEDGEMENT.}

The authors thankful to the anonymous reviewers for their valuable suggestions to improve the quality of this article. Gaddam.Chandra Mohan thanks the Director, Dept .of Technical Education, Andhra Pradesh for sponsoring him for PhD under AICTE - QIP(poly ) scheme. The support of the Director, NITTTR, and Chandigarh is acknowledged.

\section{REFERENCES}

[1] Keller, D.U.J.; Weber, F.M.; Seemann, G.; Dö ssel, O.; Ranking the Influence of Tissue Conductivities on Forward-Calculated ECGs Biomedical Engineering, IEEE Transactions on Volume: 57 , Issue: 7 Publication Year: 2010, Page(s): 1568 - 1576.

[2] Guofa Shou; Ling Xia; Mingfeng Jiang; Qing Wei; Feng Liu; Crozier, S.; Solving the ECG Forward Problem by Means of Standard h- and h-Hierarchical Adaptive Linear Boundary Element Method: Comparison With Two Refinement SchemesBiomedical Engineering, IEEE Transactions on Volume: 56 , Issue: 5 Publication Year: 2009, Page(s): $1454-1464$.

[3] Zhongshi Li; Yingchun Zhang; Shanan Zhu; Bin He; Comparison of Meshless FEM and Conventional FEM for Solving ECG Forward Problem: A Simulation Study Noninvasive Functional Source Imaging of the Brain and Heart and International Conference the Functional Biomedical Imaging,2007 Joint Meeting of the 6th International Symposium NFSI-ICFBI 2007,. Publication Year: 2007 , Page(s): 274 275.

[4] Hu Peng; Angning Yu; Qiang Chen; Huanqing Feng; Studies on New Schemes for ECG Forward Problem Simulations Engineering in Medicine and Biology Society, 2005. 27th Annual International Conference of the IEEE-EMBS 2005. Publication Year: 2005 , Page(s): 7190 - 7193.

[5] Geneser, S.E.; Seungkeol Choe; Kirby, R.M.; MacLeod, R.S.; The Influence of Stochastic Organ Conductivity in 2D ECG Forward Modeling: A Stochastic Finite Element Study Engineering in Medicine and Biology Society, 2005. 27th Annual International Conference of the IEEE-EMBS 2005. Publication Year: 2005, Page(s): $5528-5531$.

[6] Buist, M.L.; Smith, N.P.; Pullan, A.J.; Cardiac Electromechanics and the Forward/Inverse Problems of Electrocardiology IEEE Engineering in Medicine and Biology Society, 2005. -27th Annual International Conference of the EMBS 2005. Publication Year: 2005, Page(s): 7198 - 7200.

[7] Babaeizadeh, S.; Brooks, D.H.; Isaacson, D.; A 3-D boundary element solution to the forward problem of electrical impedance tomography IEEE Engineering in Medicine and Biology Society, 2004. 26th Annual International Conference of the IEMBS '04. Volume: 1 Publication Year: 2004, Page(s): 960 - 963.

[8] Cheng, L.K.; Buist, M.L.; Sands, G.B.; Pullan, A.J.; Interpretation of ECG signals through forward and inverse modelling Engineering in Medicine and Biology, 2002. 24th Annual Conference and the Annual Fall Meeting of the Biomedical Engineering Society Proceedings of the Second Joint EMBS/BMES Conference, 2002. Volume: 2 Publication Year: 2002, Page(s): 1389 - 1390 vol.2.

[9] Franz, T.; Schneider, F.R.; Dossel, O.; Forward solution of chaotic myocardial activation Computers in Cardiology 1999 Publication Year: 1999 , Page(s): 117 - 120. 
[10] Gulrajani, R.M.; The forward and inverse problems of electrocardiography IEEE

[11] Engineering in Medicine and Biology Magazine, Volume: 17, Issue: 5 Publication Year: 1998 , Page(s): $84-101,122$.

[12] Ferguson, A.S.; Stroink, G.; Factors affecting the accuracy of the boundary element method in the forward problem. I. Calculating surface potentials IEEE Transactions on Biomedical Engineering, Volume: 44 , Issue: 11 Publication Year: 1997 , Page(s): 1139 - 1155.

[13] Gulrajani, R.M.; The forward problem of electrocardiography: from heart models to body surface potentials Proceedings of the 19th Annual International Conference of the IEEE Engineering in Medicine and Biology Society, 1997. Volume: 6 Publication Year: 1997, Page(s): 2604 - 2609 vol.6. 